FACTA UNIVERSITATIS (NIŠ)

Ser. Math. Inform. Vol. 32, No 1 (2017), 11-29

DOI:10.22190/FUMI1701011V

\title{
VISUALIZATION OF LINES OF CURVATURE ON QUADRATIC SURFACES
}

\author{
Vesna Veličković
}

\begin{abstract}
In this paper, we give a complete survey of the lines of curvature on quadratic surfaces, and, more generally, on tangent surfaces, general cylinders and cones. We also apply our own software to the graphical representation of all the results we present.

Keywords: Curvature; quadratic surfaces; tangent surfaces; graphical representation.
\end{abstract}

\section{Introduction}

Quadratic surfaces or quadrics are traditionally studied in lectures on linear algebra and analytic geometry, and multi-variable analysis and vector analysis. They also provide interesting examples for the study in the differential geometry of non-trivial lines of curvature.

A curve on a surface whose tangent at each point is in a principal direction at that point is called a line of curvature. Some differential geometrical properties of those lines on parametric surfaces are discussed in [8] and on implicit surfaces in $[22,2]$. A good overview of lines of curvatures and their behavior near umbilical points and principal cycles and in the neighborhood of critical points is given in [20]. There are papers that extract lines of curvature from noisy point clouds, or construct surfaces by lines of curvature $[9,11,1,7]$.

In this paper we study the lines of curvature on quadratic surfaces. For cylinders and cones we consider more general cases, general cylinders and general cones. We also deal with tangent surfaces, since the computation is similar.

Visualization strongly supports the understanding of mathematical concepts. We developed our own software to visualize all the presented results. To be able to represent lines precisely, we use line graphics. In this approach surfaces are represented by families of lines on them, without polygon mesh. Lines are given by their parametric representation. In this paper we determine the mathematical expressions for the lines of curvature on quadratic surfaces and illustrate the obtained

Received December 03., 2016.; Accepted March 09., 2017.

2010 Mathematics Subject Classification. Primary 53A05; Secondary 00A66, 68U05 
results. All the geometrical figures in this paper have been created by our software package.

\section{Line Graphics Approach}

The main stream to represent surfaces in modern computer graphics is their approximation by the faces of polyhedra, mainly by triangulation or rectangulation, called polygon mesh. Therefore, we do not see the original surface but we do see the approximation of the surfaces.

A problem arises in the representation of curves on such surfaces. A graphical representation of a curve can be fairly precise. Then, it appears as if a curve is not really on the surface, it looks like it floats around the surface. Another approach is to approximate the curve so that it lies on the corresponding polyhedron. But then, the curve is not smooth and differs very much from the original curve.

This issue is of special interest for us, because we want to visualize lines of curvature on quadratic surfaces. The visualization of curves on surfaces plays an important role in differential geometry. As an example we mention asymptotic and geodesic lines, lines of curvature, level lines, lines of self-intersection, or the lines of intersection of surfaces.

This is why we use line graphics, and represent surfaces by families of lines on them. Since we do not make any approximation of surfaces, the curves on the surfaces can be arbitrarily accurate. The curves look smooth and they are of printing quality. The size of figures can be measured in meters without loosing quality.

The representation of a surface by line graphics is not the same as by wire models. We take into account the visibility of points. The visibility of a point is tested with respect to the surface to which it belongs, but also with respect to the other surfaces in the scene. Furthermore, the surface includes a contour line. By a contour point of a surface we mean a point at which the surface normal vector is orthogonal to the projection ray. The contour line of a surface is the set of all its contour points. Without its contour line, a surface would appear to be unfinished.

Surfaces and curves are given by their parametric representations. The visibility of points is tested analytically. Contour points are also computed analytically. We derive the corresponding formulae for each class of surfaces we want to represent. The major work in line graphics is the derivation of those formulae. Once we have obtained the formulae, we can draw curves precisely, up to the limitations of the real numbers in the programming language. Nevertheless, if the computation is complicated, the accuracy may depend on numerical errors, which can show if the figure is too large. On the other hand, visual representations can show whether the mathematics is correct.

The line graphics approach was first introduced by Endl $([3,4])$ in the late 1980's for some elementary geometrical bodies such as Platonic bodies, cones, cylinders or spheres. Starting shortly afterwards, Malkowsky used the same approach for many 
topics in classical differential geometry ([14]). Later several extensions were made in different fields, for instance in functional analysis, topology, physics, chemistry and the engineering sciences $([5,6,12,13,16,17,18,15,19,21])$.

\section{Mathematical Background}

A general definition of a quadratic surface in $\mathbb{R}^{3}$ in normal form is

$$
(\vec{x})^{T} \cdot A \cdot \vec{x}+\vec{b} \cdot \vec{x}+d=0,
$$

where $\vec{x}=(x, y, z)^{T}$ is a column vector, $\vec{b}=\left(0,0, b_{3}\right), b_{3} \in\{0,-1\}$ is a row vector, $d \in\{-1,0,1\}$, and the diagonal matrix $A$ is

$$
A=\left(\begin{array}{ccc}
a_{1} & 0 & 0 \\
0 & a_{2} & 0 \\
0 & 0 & a_{3}
\end{array}\right) .
$$

For various entries in the matrix $A$, and value of $b_{3}$ and $d$, we get different quadratic surfaces. There are nine real nontrivial quadratic surfaces.

For example, for $a_{1}=\frac{1}{a^{2}}, a_{2}=\frac{1}{b^{2}}, a_{3}=\frac{1}{c^{2}}, a, b, c \in \mathbb{R} \backslash\{0\}, b_{3}=0$ and $d=-1$, we have the ellipsoid

It is easy to verify that

$$
\frac{x^{2}}{a^{2}}+\frac{y^{2}}{b^{2}}+\frac{z^{2}}{c^{2}}=1
$$

$$
\vec{x}\left(u^{i}\right)=\left\{\frac{1}{\sqrt{a_{1}}} \cos u^{1} \cos u^{2}, \frac{1}{\sqrt{a_{2}}} \cos u^{1} \sin u^{2}, \frac{1}{\sqrt{a_{3}}} \sin u^{1}\right\}
$$

is a parametric representation of the ellipsoid for $\left(u^{1}, u^{2}\right) \in\left(-\frac{\pi}{2}, \frac{\pi}{2}\right) \times(0,2 \pi)$.

Similarly, the hyperboloid of one sheet $\left(a_{1}=\frac{1}{a^{2}}, a_{2}=\frac{1}{b^{2}}, a_{3}=-\frac{1}{c^{2}}, b_{3}=0\right.$ and $d=-1)$ has a parametric representation

$$
\vec{x}\left(u^{i}\right)=\left\{\frac{1}{\sqrt{a_{1}}} \cosh u^{1} \cos u^{2}, \frac{1}{\sqrt{a_{2}}} \cosh u^{1} \sin u^{2}, \frac{1}{\sqrt{-a_{3}}} \sinh u^{1}\right\}
$$

for $\left(u^{1}, u^{2}\right) \in \mathbb{R} \times(0,2 \pi)$.

We can get the parametric representations of the other quadratic surfaces in the similar way, except for parabolic cylinder $\left(a_{1}=\frac{1}{a^{2}}, a_{2}=0, a_{3}=0, b_{3}=-1\right.$ and $d=0)$ that has a parametric representation

$$
\vec{x}\left(u^{i}\right)=\left\{\frac{1}{\sqrt{a_{1}}} u^{1}, u^{2},\left(u^{1}\right)^{2}\right\}, \quad\left(\left(u^{1}, u^{2}\right) \in \mathbb{R} \times(0,2 \pi)\right)
$$

and for the hyperbolic paraboloid $\left(a_{1}=\frac{1}{a^{2}}, a_{2}=-\frac{1}{b^{2}}, a_{3}=0, b_{3}=-1\right.$ and $\left.d=0\right)$ with

$$
\left.\vec{x}\left(u^{i}\right)=\left\{\frac{1}{\sqrt{a_{1}}} u^{1}, \frac{1}{\sqrt{-a_{2}}} u^{2},\left(u^{1}\right)^{2}-\left(u^{2}\right)^{2}\right\}, \quad\left(\left(u^{1}, u^{2}\right) \in \mathbb{R} \times \mathbb{R}\right)\right) .
$$


Now we give a short survey of the standard background from differential geometry, and introduce the necessary notations.

Let $D \subset \mathbb{R}^{2}$ be a domain and $S$ be a surface in $\mathbb{R}^{3}$ with a parametric representation

$$
\vec{x}\left(u^{i}\right)=\vec{x}\left(u^{1}, u^{2}\right)=\left(x^{1}\left(u^{1}, u^{2}\right), x^{2}\left(u^{1}, u^{2}\right), x^{3}\left(u^{1}, u^{2}\right)\right) \quad\left(\left(u^{1}, u^{2}\right) \in D\right) .
$$

We always assume that $S$ is of class $\mathcal{C}^{r}(D)$ for some $r \geqslant 1$, that is, the functions $x^{k}(k=1,2,3)$ in $(3.2)$ have continuous derivatives of order $r$ on $D$, where $r$ is chosen according to need. Furthermore, we assume that the vector-valued functions $\vec{x}_{k}=\partial \vec{x} / \partial u^{k} \quad(k=1,2)$ are linearly independent on $D$. Thus the tangent plane to $S$ spanned by the vectors $\vec{x}_{1}\left(u^{i}\right)$ and $\vec{x}_{2}\left(u^{i}\right)$ exists at every point of $S$. The vectors

$$
\vec{N}\left(u^{i}\right)=\frac{\vec{x}_{1}\left(u^{i}\right) \times \vec{x}_{2}\left(u^{i}\right)}{\left\|\vec{x}_{1}\left(u^{i}\right) \times \vec{x}_{2}\left(u^{i}\right)\right\|} \quad\left(\left(u^{1}, u^{2}\right) \in D\right)
$$

are called surface normal vectors of $S$ (Figure 3.1).

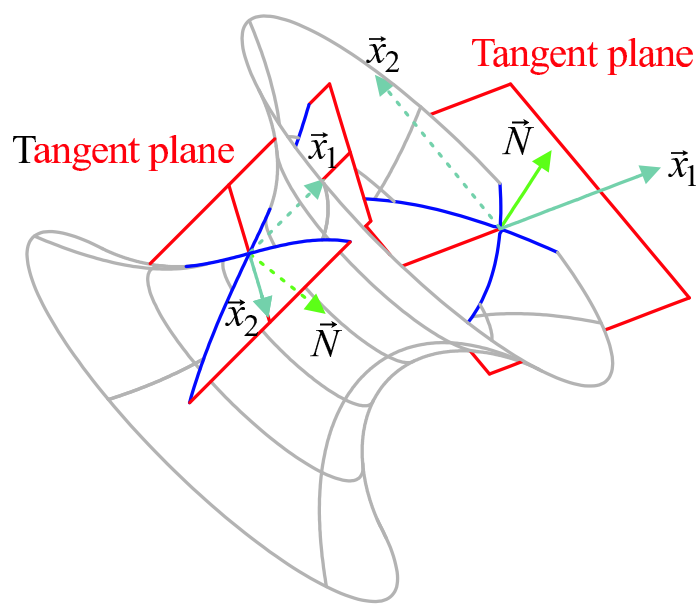

FIG. 3.1: The vectors $\vec{x}_{k}$, tangent planes and surface normal vectors

The first and second fundamental coefficients of a surface $S$ are denoted by $g_{i k}$ and $L_{i k}(i, k=1,2)$, that is, if $S$ has a parametric representation (3.2), then

$$
g_{i k}\left(u^{j}\right)=\vec{x}_{i}\left(u^{j}\right) \bullet \vec{x}_{k}\left(u^{j}\right) \text { and } L_{i k}\left(u^{j}\right)=\vec{N}\left(u^{j}\right) \bullet \vec{x}_{i k}\left(u^{j}\right) \text { where } \vec{x}_{i k}=\frac{\partial^{2} \vec{x}}{\partial u^{i} \partial u^{k}}
$$

for $i, k=1,2$; we also write

$$
g\left(u^{j}\right)=g_{11}\left(u^{j}\right) g_{22}\left(u^{j}\right)-g_{12}^{2}\left(u^{j}\right) \text { and } L\left(u^{j}\right)=L_{11}\left(u^{j}\right) L_{22}\left(u^{j}\right)-L_{12}^{2}\left(u^{j}\right) .
$$

It follows from $g\left(u^{j}\right)=\left(\vec{x}_{1}\left(u^{j}\right) \times \vec{x}_{2}\left(u^{j}\right)\right)^{2}$ that $g\left(u^{j}\right)>0$ for all $\left(u^{1}, u^{2}\right) \in D$. 
Let $S$ be a surface with a parametric representation (3.2) and $\gamma$ be a curve on $S$ given by $\vec{x}(s)=\vec{x}\left(u^{i}(s)\right)$ for $s \in I \subset \mathbb{R}$ where $s$ is the arc length along $\gamma$. We denote derivatives with respect to $s$ by a dot. The vector of curvature $\ddot{\vec{x}}(s)$ of $\gamma$ at $s$ is split into two components (left on the figure 3.2)

$$
\ddot{\vec{x}}(s)=\kappa_{n}(s) \vec{N}\left(u^{i}(s)\right)+\kappa_{g}(s) \vec{t}\left(u^{i}(s)\right) \text { where } \vec{t}\left(u^{i}(s)\right)=\vec{N}\left(u^{i}(s)\right) \times \dot{\vec{x}}(s) .
$$

The values $\kappa_{n}(s)$ and $\kappa_{g}(s)$ are called the normal and geodesic curvature of $\gamma$ at $s$.

At any point $P$ on a surface, there corresponds one and only one value of the normal curvature to any direction ([10, Satz 5.1,p. 46]). The extreme values of the normal curvature are called principal curvatures, and the directions corresponding to the principal curvatures are called principal directions (right on the figure 3.2). It is well known that at every point of a surface there are two orthogonal principal directions.

\section{Plane spanned by $\vec{t}$ and $\vec{N}$}

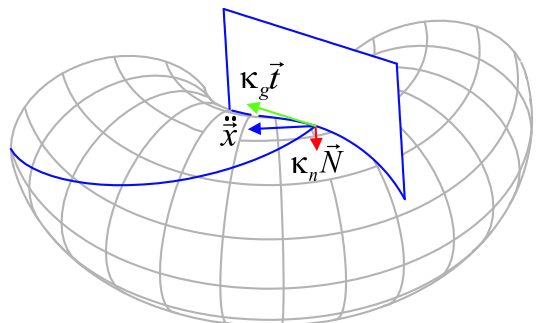

Curve $\vec{x}(s)$

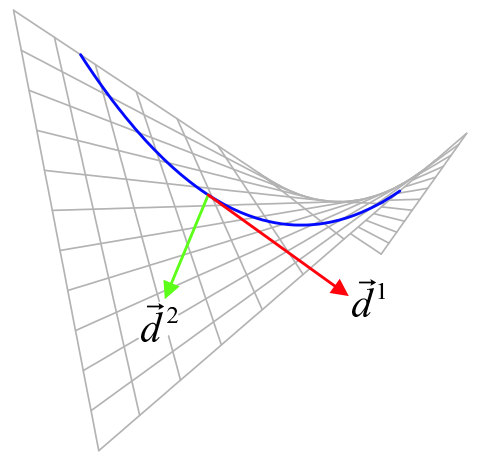

FIG. 3.2: Left: The components of a vector of curvature;

Right: Principal directions at a point

A curve on a surface which has the property that its tangent at each of its points $P$ coincides with a principal direction at $P$ is called line of curvature (Figure 3.3).

The next result is well known and useful to determine the lines of curvature.

Proposition 3.1. ([10, (5.12)])

The lines of curvature are given by the solutions of the differential equation

$$
\operatorname{det}\left(\begin{array}{ll}
L_{11} d u^{1}+L_{12} d u^{2} & g_{11} d u^{1}+g_{12} d u^{2} \\
L_{21} d u^{1}+L_{22} d u^{2} & g_{21} d u^{1}+g_{22} d u^{2}
\end{array}\right)=0
$$




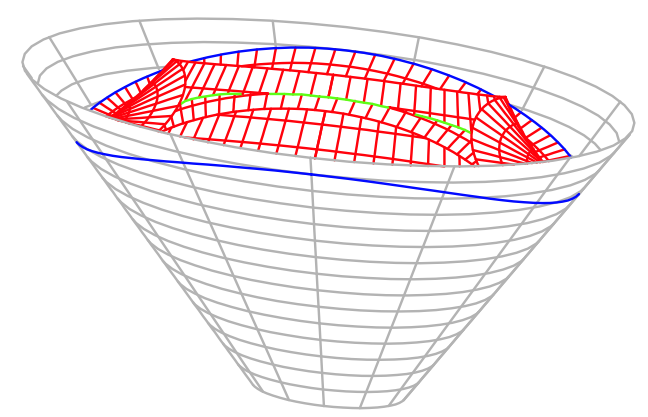

FIG. 3.3: Surface generated by the surface normal vectors along a line of curvature on an elliptical cone

\section{Lines of Curvature on Tangent Surfaces, General Cylinders and General Cones}

In this section, we determine and represent the lines of curvature on the quadratic surfaces that are cylinders or cones. Even more, we consider general cylinders and general cones. Furthermore, we determine the lines of curvature on tangent surfaces, since the computation is similar. General cylinders, general cones and tangent surface are special kinds of ruled surfaces. There are shown on Figure 4.1.

Let $\gamma$ be a curve with a parametric representation $\vec{y}(t)\left(t \in I_{1} \subset \mathbb{R}\right)$. Furthermore, for every $t \in I_{1}$, let $\vec{z}(t)$ be a unit vector. Then a ruled surface is generated by the movement along $\gamma$ of the straight lines defined by the vectors $\vec{z}(t)$. We put $u^{1}=t$ and obtain the following parametric representation for a ruled surface (Figures 4.2 and 4.3 )

$$
\vec{x}\left(u^{i}\right)=\vec{y}\left(u^{1}\right)+u^{2} \vec{z}\left(u^{1}\right), \quad\left(\left(u^{1}, u^{2}\right) \in R=I_{1} \times I_{2}\right) .
$$

It follows that

$$
\begin{aligned}
\vec{x}_{1}\left(u^{i}\right)=\vec{y}^{\prime}\left(u^{1}\right)+u^{2} \vec{z}^{\prime}\left(u^{1}\right), & \vec{x}_{2}\left(u^{i}\right)=\vec{z}\left(u^{1}\right), \\
\vec{x}_{11}\left(u^{i}\right)=\vec{y}^{\prime \prime}\left(u^{1}\right)+u^{2} \vec{z}^{\prime \prime}\left(u^{1}\right), & \vec{x}_{12}\left(u^{i}\right)=\vec{z}^{\prime}\left(u^{1}\right) \quad \text { and } \vec{x}_{22}\left(u^{i}\right)=\overrightarrow{0},
\end{aligned}
$$

and so, since $\vec{z}\left(u^{1}\right) \bullet \vec{z}^{\prime}\left(u^{1}\right)=0$ for all $u^{1} \in I_{1}$,

$$
\begin{gathered}
g_{11}\left(u^{i}\right)=\left(\vec{y}^{\prime}\left(u^{1}\right)\right)^{2}+2 u^{2} \vec{y}^{\prime}\left(u^{1}\right) \bullet \vec{z}^{\prime}\left(u^{1}\right)+\left(u^{2}\right)^{2}\left(\vec{z}^{\prime}\left(u^{1}\right)\right)^{2} \\
g_{12}\left(u^{i}\right)=\vec{y}^{\prime}\left(u^{1}\right) \bullet \vec{z}\left(u^{1}\right), \quad g_{22}\left(u^{i}\right)=1 \\
g\left(u^{i}\right)=\left(\vec{y}^{\prime}\left(u^{1}\right)\right)^{2}+2 u^{2} \vec{y}^{\prime}\left(u^{1}\right) \bullet \vec{z}^{\prime}\left(u^{1}\right)+\left(u^{2}\right)^{2}\left(\vec{z}^{\prime}\left(u^{1}\right)\right)^{2}-\left(\vec{y}^{\prime}\left(u^{1}\right) \bullet \vec{z}\left(u^{1}\right)\right)^{2} \\
L_{11}\left(u^{i}\right)=\vec{x}_{11}\left(u^{i}\right) \bullet \frac{\left(\vec{x}_{1}\left(u^{i}\right) \times \vec{x}_{2}\left(u^{i}\right)\right)}{\sqrt{g\left(u^{i}\right)}} \\
L_{12}\left(u^{i}\right)=\vec{z}^{\prime}\left(u^{1}\right) \bullet \frac{\left(\vec{x}_{1}\left(u^{i}\right) \times \vec{x}_{2}\left(u^{i}\right)\right)}{\sqrt{g\left(u^{i}\right)}} \\
L_{22}\left(u^{i}\right)=0 \text { and } L\left(u^{i}\right)=-L_{12}^{2}\left(u^{i}\right) .
\end{gathered}
$$




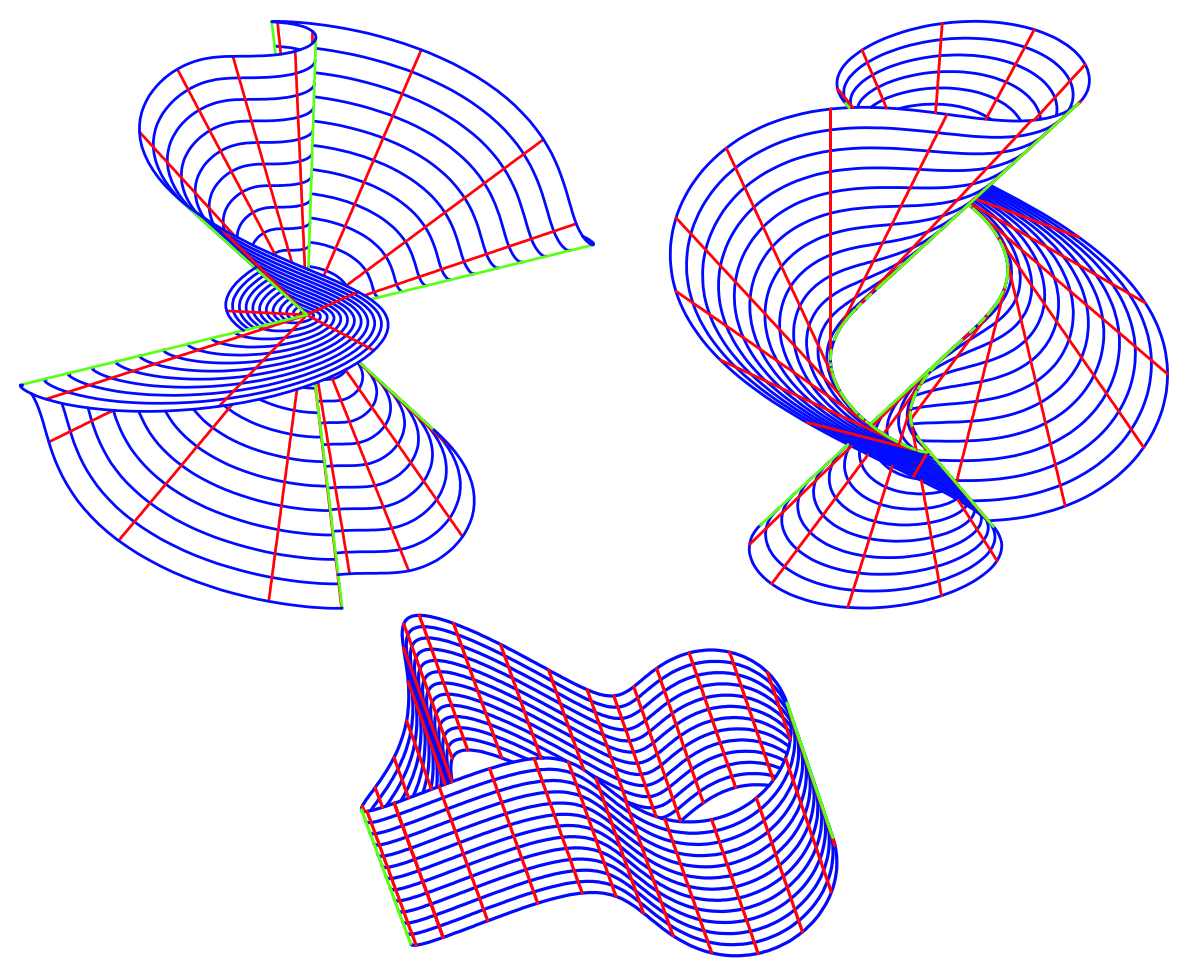

FIG. 4.1: Left: A general cone; Right: A tangent surface; Down: A general cylinder

It is easy to see that, in the special case of general cylinders, cones and tangent surfaces, we have

$$
\vec{y}^{\prime}\left(u^{1}\right) \bullet\left(\vec{z}\left(u^{1}\right) \times \vec{z}^{\prime}\left(u^{1}\right)\right)=0 \text { for all }\left(u^{1}, u^{2}\right) \in R,
$$

and the differential equation (3.3) for the lines of curvature reduces to

$$
\begin{gathered}
L_{11}\left(u^{i}\right) d u^{1}\left(g_{12}\left(u^{i}\right) d u^{1}+g_{22}\left(u^{i}\right) d u^{2}\right) \\
=L_{11}\left(u^{i}\right) d u^{1}\left(\left(\vec{y}^{\prime}\left(u^{1}\right) \bullet \vec{z}\left(u^{1}\right)\right) d u^{1}+d u^{2}\right)=0 .
\end{gathered}
$$

Since $L_{11}\left(u^{i}\right) \neq 0$ except for the trivial case of a plane, the $u^{2}$-lines are lines of curvature. The second family of lines of curvature is given by

$$
u^{2}\left(u^{1}\right)=-\int \vec{y}^{\prime}\left(u^{1}\right) \bullet \vec{z}\left(u^{1}\right) d u^{1} .
$$

If the surface is a general cylinder, then $\vec{z}\left(u^{1}\right)=\vec{c}$, a constant vector. We obtain from (4.2)

$$
u^{2}\left(u^{1}\right)=-\vec{c} \bullet \vec{y}\left(u^{1}\right)+d \text { where } d \in \mathbb{R} \text { is a constant (Figure 4.4). }
$$




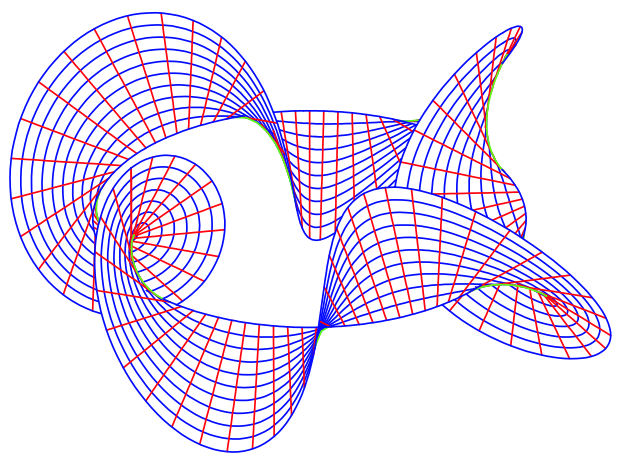

FIG. 4.2: A ruled surface with $y\left(u^{1}\right)=4 \cos u^{1}$ and $z\left(u^{1}\right)=\left(\cos u^{1} \sin 4 u^{1}, \sin u^{1} \sin 4 u^{1}, \cos 4 u^{1}\right)$ for $\left(u^{1}, u^{2}\right) \in[0,2 \pi] \times[0,3]$

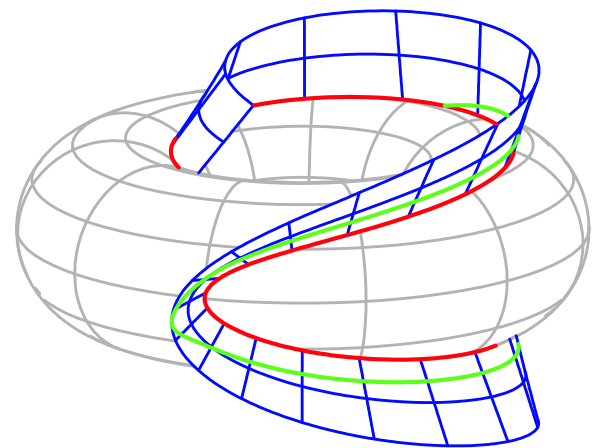

FIG. 4.3: Representation of the normal curvature along a curve $\vec{x}\left(u^{i}(t)\right)$ on a torus as $\vec{y}(t)=\vec{x}\left(u^{i(t)}\right)-\kappa_{n}\left(u^{i}(t)\right) \cdot \vec{N}\left(u^{i}(t)\right)$

If the surface is a general cone, then let $\vec{c} \neq \vec{y}\left(u^{1}\right)$ denote the position vector of the point of intersection of all straight lines of the ruled surface. We may assume $\vec{c}=\overrightarrow{0}$, for otherwise we apply a linear transformation of the coordinate system. Putting $\vec{z}\left(u^{1}\right)=\vec{y}\left(u^{1}\right) /\left\|\vec{y}\left(u^{1}\right)\right\|$, we obtain from (4.2)

$$
u^{2}\left(u^{1}\right)=-\int \frac{\vec{y}^{\prime}\left(u^{1}\right) \bullet \vec{y}\left(u^{1}\right)}{\left\|\vec{y}\left(u^{1}\right)\right\|} d u^{1}=\left\|\vec{y}\left(u^{1}\right)\right\|+d
$$

where $d \in \mathbb{R}$ is a constant (Figure 4.5).

If the surface is a tangent surface, then $\vec{z}\left(u^{1}\right)=\vec{y}^{\prime}\left(u^{1}\right) /\left\|\vec{y}^{\prime}\left(u^{1}\right)\right\|$. We obtain from (4.2)

$$
u^{2}\left(u^{1}\right)=-\int \frac{\vec{y}^{\prime}\left(u^{1}\right) \bullet \vec{y}^{\prime}\left(u^{1}\right)}{\left\|\vec{y}^{\prime}\left(u^{1}\right)\right\|} d u^{1}=-\int\left\|\vec{y}^{\prime}\left(u^{1}\right)\right\| d u^{1} \text { (Figure 4.6), }
$$

that is, $u^{2}$ is the arc length along the curve $\gamma$ given by $\vec{y}\left(u^{1}\right)$. 


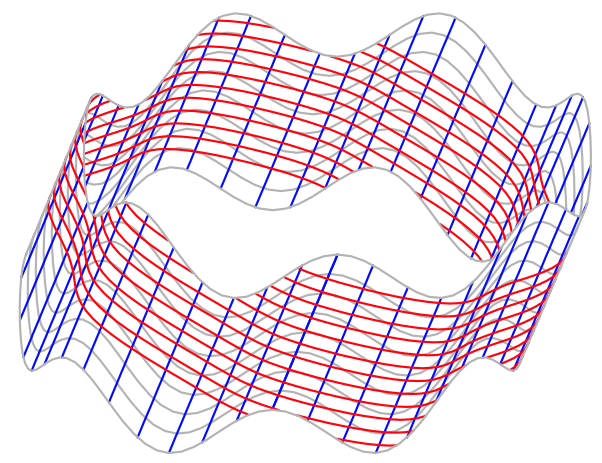

FiG. 4.4: Lines of curvature on a general cylinder
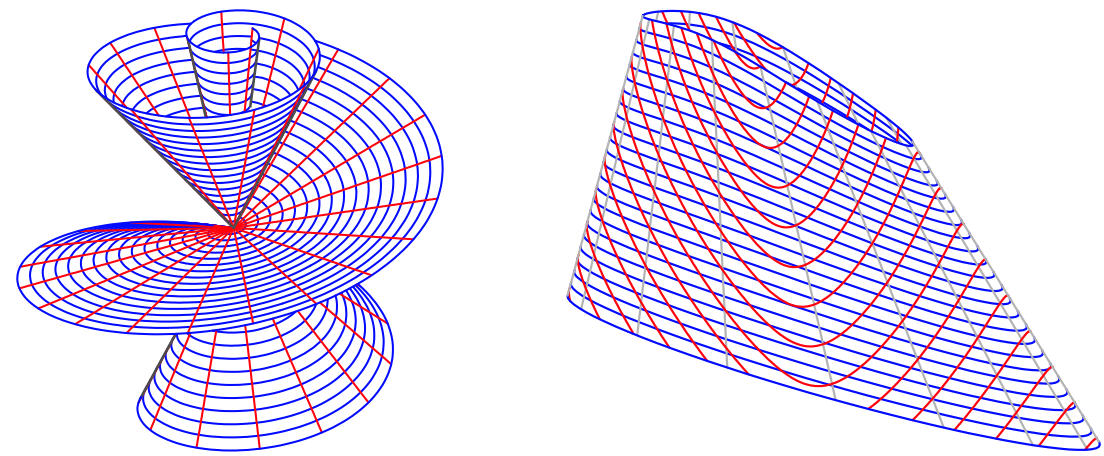

FIG. 4.5: Lines of curvature on two general cones

Left: Lines of curvatures coincide with parameter lines Right: General case

The following well-known result is useful for finding the lines of curvature on quadratic cylinders.

Proposition 4.1. ([10, Problem 5.7, p. 41])

The lines of curvature of a surface coincide with its parameter lines if and only if $g_{12} \equiv 0$ and $L_{12} \equiv 0$.

Example 4.1. Let $a$ and $b$ be positive real numbers. For the elliptic, parabolic and hyperbolic cylinder with parametric representations

$$
\begin{array}{cl}
\vec{x}\left(u^{i}\right)=\left(a \cos u^{2}, b \sin u^{2}, u^{1}\right) & \left(\left(u^{1}, u^{2}\right) \in D=\mathbb{R} \times(0,2 \pi)\right), \\
\vec{x}\left(u^{i}\right)=\left(a \cdot u^{1}, u^{2},\left(u^{1}\right)^{2}\right) & \left(\left(u^{1}, u^{2}\right) \in D=\mathbb{R}^{2}\right), \\
\vec{x}\left(u^{i}\right)=\left( \pm a \cosh u^{2}, b \sinh u^{2}, u^{1}\right) & \left(\left(u^{1}, u^{2}\right) \in D=\mathbb{R}^{2}\right),
\end{array}
$$

respectively, we have $g_{12} \equiv 0$ and $L_{12} \equiv 0$. Hence the parameter lines are lines of curvature by Proposition 4.1 . 


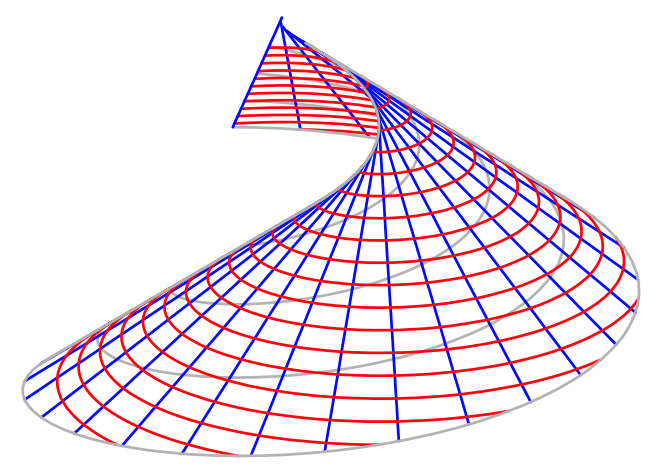

Fig. 4.6: Lines of curvature on a tangent surface

We close this section with giving the lines of curvature on elliptic and hyperbolic cones.

Example 4.2. Let $a$ and $b$ be positive real numbers.

(a) We consider the elliptic cone with a parametric representation

$$
\vec{x}\left(u^{i}\right)=\left(a u^{1} \cos u^{2}, b u^{1} \sin u^{2}, u^{1}\right)\left(\left(u^{1}, u^{2}\right) \in D=(0, \infty) \times(0,2 \pi)\right) .
$$

Putting $u^{* 1}=u^{2}, \vec{y}^{*}\left(u^{* 1}\right)=\left(a \cos u^{* 1}, b \sin u^{* 1}, 1\right)$ and $u^{* 2}=\left(1-u^{1}\right)\left\|\vec{y}^{*}\left(u^{2}\right)\right\|$, we obtain the parametric representation

$$
\vec{x}^{*}\left(u^{* i}\right)=\left(1-\frac{u^{* 2}}{\left\|\vec{y}^{*}\left(u^{* 1}\right)\right\|}\right) \vec{y}^{*}\left(u^{* 1}\right)
$$

for the elliptic cone. The lines of curvature are the $u^{* 2}$-lines with respect to the parametric representation (4.7), that is, the $u^{1}$-lines with respect to the parametric representation (4.6), and by (4.4), the curves given by $u^{* 2}\left(u^{* 1}\right)=\left\|\vec{y}^{*}\left(u^{* 1}\right)\right\|+d$ where $d \in \mathbb{R}$ is a constant. We put $k=-d$ and obtain from the transformation formulae for the parameters (Figure 4.7)

$$
\begin{aligned}
u^{1}\left(u^{2}\right) & =1-\frac{u^{* 2}}{\left\|\vec{y}^{*}\left(u^{* 1}\right)\right\|}=1-\left(1+\frac{d}{\left\|\vec{y}^{*}\left(u^{* 1}\right)\right\|}\right) \\
& =\frac{k}{\sqrt{a^{2} \cos ^{2} u^{2}+b^{2} \sin ^{2} u^{2}+1}}, \text { for } k>0, \text { since } u^{1}>0 .
\end{aligned}
$$

(b) We consider the hyperbolic cone with a parametric representation

$$
\vec{x}\left(u^{i}\right)=\left( \pm a u^{1} \cosh u^{2}, b u^{1} \sinh u^{2}, u^{1}\right)\left(\left(u^{1}, u^{2}\right) \in D+(0, \infty) \times \mathbb{R}\right) .
$$

As in Part (a), we obtain that the lines of curvature are the $u^{1}$-lines and the lines given by (Figure 4.8)

$$
u^{1}\left(u^{2}\right)=\frac{k}{\sqrt{a^{2} \cosh ^{2} u^{2}+b^{2} \sinh ^{2} u^{2}+1}} \text { for } k>0 .
$$




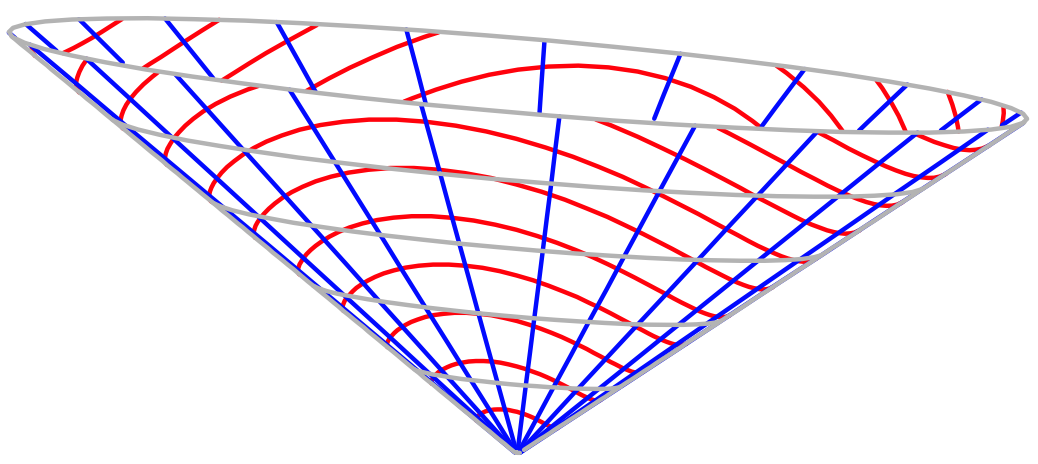

FIG. 4.7: Lines of curvature on an elliptic cone

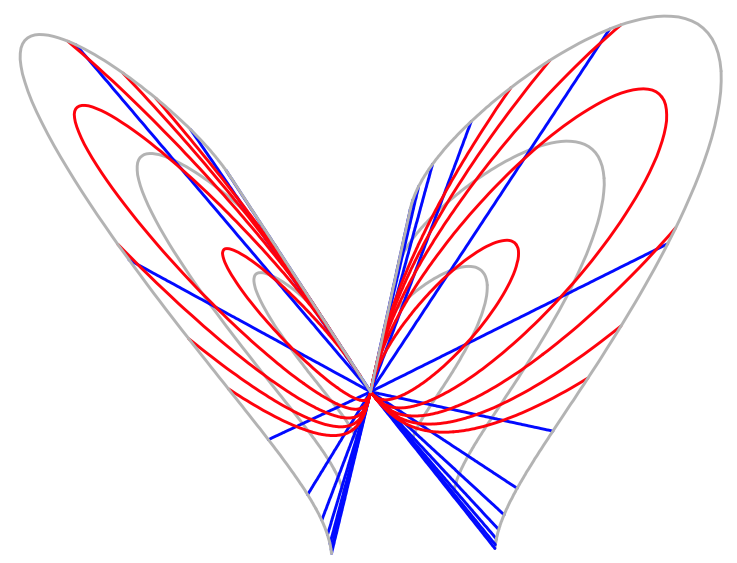

FIG. 4.8: Lines of curvature on a hyperbolic cone

\section{Lines of Curvature on Hyperbolic Paraboloids}

In this section, we determine and represent the lines of curvature on hyperbolic paraboloids.

Example 5.1. Let $a$ and $b$ be positive real numbers. Here we consider the hyperbolic paraboloid with a parametric representation

$$
\vec{x}\left(u^{i}\right)=\left(a u^{1}, b u^{2},\left(u^{1}\right)^{2}-\left(u^{2}\right)^{2}\right)\left(\left(u^{1}, u^{2}\right) \in D=\mathbb{R}^{2}\right) .
$$

We introduce new parameters $u^{* i}(i=1,2)$ by

$$
u^{1}=u^{* 1}+u^{* 2} \text { and } u^{2}=u^{* 1}-u^{* 2} .
$$

Then we may represent the hyperbolic paraboloid by

$$
\vec{x}^{*}\left(u^{* i}\right)=\left(a\left(u^{* 1}+u^{* 2}\right), b\left(u^{* 1}-u^{* 2}\right), 4 u^{* 1} u^{* 2}\right)\left(\left(u^{* 1}, u^{* 2}\right) \in \mathbb{R}^{2}\right) .
$$


The fundamental coefficients are given by

$$
\begin{gathered}
g_{11}^{*}\left(u^{* i}\right)=a^{2}+b^{2}+16\left(u^{* 2}\right)^{2}, g_{12}^{*}\left(u^{* i}\right)=a^{2}-b^{2}+16 u^{* 1} u^{* 2}, \\
g_{22}^{*}\left(u^{* i}\right)=a^{2}+b^{2}+16\left(u^{* 1}\right)^{2} \\
L_{11}^{*}\left(u^{* i}\right)=L_{22}^{*}\left(u^{* i}\right)=0 \text { and } L_{12}^{*}\left(u^{* i}\right)=-8 a b .
\end{gathered}
$$

We omit the arguments $u^{* i}$. Then the differential equation (3.3) reduces to

$$
L_{12}^{*}\left(d u^{* 2}\left(g_{12}^{*} d u^{* 1}+g_{22}^{*} d u^{* 2}\right)-d u^{* 1}\left(g_{11}^{*} d u^{* 1}+g_{12}^{*} d u^{* 2}\right)\right)=0 .
$$

Since $L_{12}^{*} \neq 0$ on $\mathbb{R}^{2}$, equation (5.3) is equivalent to

$$
g_{22}^{*}\left(d u^{* 2}\right)^{2}+\left(g_{12}^{*}-g_{12}^{*}\right) d u^{* 1} d u^{* 2}-g_{11}^{*}\left(d u^{* 1}\right)^{2}=g_{22}^{*}\left(d u^{* 2}\right)^{2}-g_{11}^{*}\left(d u^{* 1}\right)^{2}=0 .
$$

Therefore we have

$$
\int \frac{d u^{* 2}}{\sqrt{1+\left(\frac{4 u^{* 2}}{\sqrt{a^{2}+b^{2}}}\right)^{2}}}= \pm \int \frac{d u^{* 1}}{\sqrt{1+\left(\frac{4 u^{* 1}}{\sqrt{a^{2}+b^{2}}}\right)^{2}}}
$$

We obtain from this

$$
\operatorname{arsinh}\left(\frac{4 u^{* 2}}{\sqrt{a^{2}+b^{2}}}\right)= \pm \operatorname{arsinh}\left(\frac{4 u^{* 1}}{\sqrt{a^{2}+b^{2}}}\right)+C \text { where } C \in \mathbb{R} \text { is a constant, }
$$

and, putting $c=\sinh C, u^{* 2}\left(u^{* 1}\right)= \pm u^{* 1} \sqrt{1+c^{2}}+(c / 4) \sqrt{a^{2}+b^{2}+\left(4 u^{* 1}\right)^{2}}$. Hence a parametric representation for the lines of curvature on the hyperbolic paraboloid is (Figure $5.1)$

$$
\left\{\begin{array}{l}
u^{1}(t)=\left(1 \pm \sqrt{1+c^{2}}\right) t+\frac{c}{4} \sqrt{a^{2}+b^{2}+16 t^{2}} \\
u^{2}(t)=\left(1 \mp \sqrt{1+c^{2}}\right) t-\frac{c}{4} \sqrt{a^{2}+b^{2}+16 t^{2}}
\end{array}\right\} \quad(t \in \mathbb{R}) .
$$

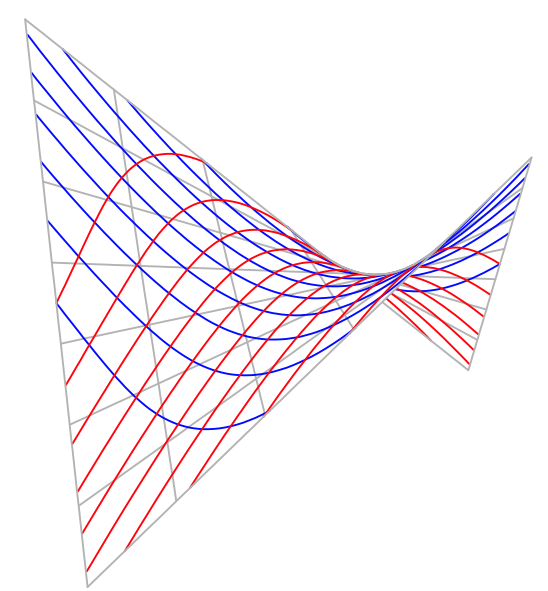

FIG. 5.1: Lines of curvature on a hyperbolic paraboloid 


\section{Triple orthogonal systems}

Triple orthogonal systems of surfaces are often useful to find the lines of curvature.

Let $\vec{y}\left(u^{1}, u^{2}, u^{3}\right)$ be of class $\mathcal{C}^{2}$, the vectors $\vec{y}_{k}=\partial \vec{y} / \partial u^{k}(k=1,2,3)$ be linearly independent, and $\vec{y}_{k} \bullet \vec{y}_{i}=0(i \neq k)$. Then the surfaces $u^{j}=$ const make up three families of mutually orthogonal surfaces, a so-called triple orthogonal system.

Example 6.1. Let

$$
\begin{array}{r}
\vec{y}\left(u^{1}, u^{2}, u^{3}\right)=\left(u^{1} \cos u^{2} \cos u^{3}, u^{1} \cos u^{2} \sin u^{3}, u^{1} \sin u^{2}\right) \\
\left(\left(u^{1}, u^{2}, u^{3}\right) \in(0, \infty) \times(-\pi / 2, \pi / 2) \times(0,2 \pi)\right) .
\end{array}
$$

For $u^{1}=r>0$, we obtain the sphere of radius $r$ centred at the origin. For $u^{2}=$ const, we obtain a cone with its vertex in the origin. For $u^{3}=$ const, we obtain a plane orthogonal to the $x^{1} x^{3}$ - plane. Furthermore, $\vec{y}$ is of class $\mathcal{C}^{2}$,

$$
\begin{aligned}
& \vec{y}_{1}\left(u^{1}, u^{2}, u^{3}\right)=\left(\cos u^{2} \cos u^{3}, \cos u^{2} \sin u^{3}, \sin u^{2}\right), \\
& \vec{y}_{2}\left(u^{1}, u^{2}, u^{3}\right)=\left(-u^{1} \sin u^{2} \cos u^{3},-u^{1} \sin u^{2} \sin u^{3}, u^{1} \cos u^{2}\right), \\
& \vec{y}_{3}\left(u^{1}, u^{2}, u^{3}\right)=\left(-u^{1} \cos u^{2} \sin u^{3}, u^{1} \cos u^{2} \cos u^{3}, 0\right),
\end{aligned}
$$

and it is easy to see that $\vec{y}_{k} \bullet \vec{y}_{i}=0$ for $i \neq k$. Consequently the surfaces are members of a triple orthogonal system (Figure 6.1).

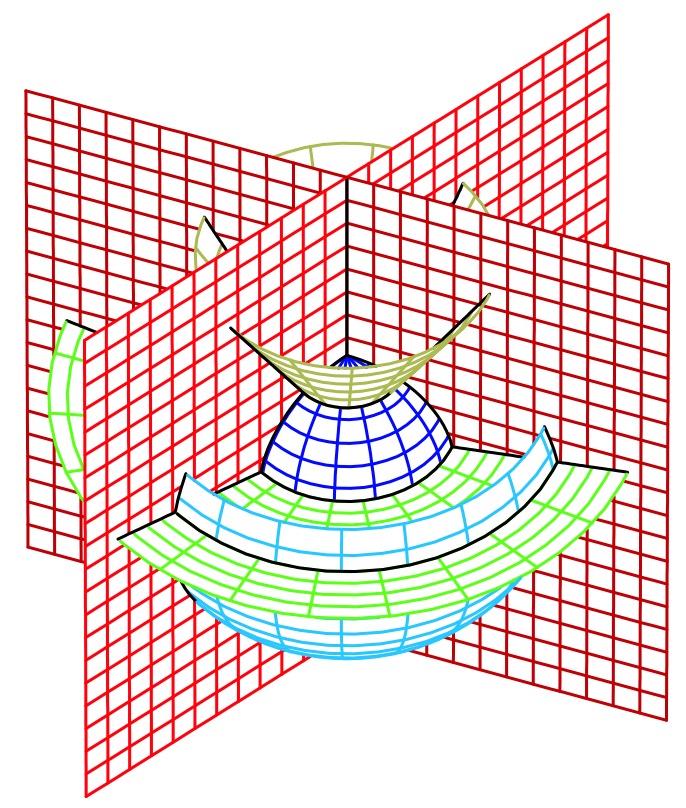

FIG. 6.1: The triple orthogonal system of Example 6.1

The following result is well known. 
Theorem 6.1. Dupin ([10, Satz 6.9, p. 63]) The surfaces of a triple orthogonal system intersect in lines of curvature.

\section{Lines of curvature on ellipsoids and hyperboloids}

In this section, we apply Dupin's theorem to find the lines of curvature on ellipsoids and hyperboloids of one and two sheets.

We need the following result.

Lemma 7.1. Let $a, b, c \in \mathbb{R}$ with $0<a^{2}<b^{2}<c^{2}$ be given. We consider the class $\mathcal{S}$ of surfaces given by the equations

$$
g(\lambda)=\frac{\left(x^{1}\right)^{2}}{a^{2}-\lambda}+\frac{\left(x^{2}\right)^{2}}{b^{2}-\lambda}+\frac{\left(x^{3}\right)^{2}}{c^{2}-\lambda}-1=0 \quad\left(\lambda \neq a^{2}, b^{2}, c^{2}\right)
$$

If $\lambda<a^{2}$, then the surfaces are ellipsoids. If $a^{2}<\lambda<b^{2}$, then the surfaces are hyperboloids of one sheet. If $b^{2}<\lambda<c^{2}$, then the surfaces are hyperboloids of two sheets. There is one and only one surface of each family through each point $\left(x^{1}, x^{2}, x^{3}\right) \in \mathbb{R}^{3} \backslash\{0\}$. The class $\mathcal{S}$ is a triple orthogonal system.

Proof. For any fixed point $\left(x^{1}, x^{2}, x^{3}\right) \in \mathbb{R}^{3} \backslash\{0\}$, the function $g$ is continuous on $\mathbb{R} \backslash\left\{a^{2}, b^{2}, c^{2}\right\}$, and

$$
\begin{aligned}
\lim _{\lambda \rightarrow a^{2}-} g(\lambda) & =\lim _{\lambda \rightarrow b^{2}-} g(\lambda)=\lim _{\lambda \rightarrow c^{2}-} g(\lambda)=+\infty, \\
\lim _{\lambda \rightarrow a^{2}+} g(\lambda) & =\lim _{\lambda \rightarrow b^{2}+} g(\lambda)=\lim _{\lambda \rightarrow c^{2}+} g(\lambda)=-\infty, \\
\lim _{\lambda \rightarrow-\infty} g(\lambda) & =\lim _{\lambda \rightarrow+\infty} g(\lambda)=-1 .
\end{aligned}
$$

Thus there are values $\lambda_{1}, \lambda_{2}, \lambda_{3} \in \mathbb{R}$ with

$$
\lambda_{1}<a^{2}<\lambda_{2}<b^{2}<\lambda_{3}<c^{2} \text { and } g\left(\lambda_{k}\right)=0(k=1,2,3) .
$$

On the other hand, $g$ has at most three zeros, since $g(\lambda)=0$ is equivalent to a cubic equation.

The surface normal vector at a point $\left(x^{1}, x^{2}, x^{3}\right) \in \mathbb{R}^{3} \backslash\{0\}$ to the surface given by the equation $g\left(\lambda_{k}\right)=0$ has the direction

$$
\frac{1}{2} \operatorname{grad} g\left(\lambda_{k}\right)=\left(\frac{x^{1}}{a^{2}-\lambda_{k}}, \frac{x^{2}}{b^{2}-\lambda_{k}}, \frac{x^{3}}{c^{2}-\lambda_{k}}\right),
$$


and we have for $i \neq k$

$$
\begin{array}{r}
\left(\frac{x^{1}}{a^{2}-\lambda_{i}}, \frac{x^{2}}{b^{2}-\lambda_{i}}, \frac{x^{3}}{c^{2}-\lambda_{i}}\right) \bullet\left(\frac{x^{1}}{a^{2}-\lambda_{k}}, \frac{x^{2}}{b^{2}-\lambda_{k}}, \frac{x^{3}}{c^{2}-\lambda_{k}}\right) \\
=\frac{\left(x^{1}\right)^{2}}{\left(a^{2}-\lambda_{i}\right)\left(a^{2}-\lambda_{k}\right)}+\frac{\left(x^{2}\right)^{2}}{\left(b^{2}-\lambda_{i}\right)\left(b^{2}-\lambda_{k}\right)}+\frac{\left(x^{3}\right)^{2}}{\left(c^{2}-\lambda_{i}\right)\left(c^{2}-\lambda_{k}\right)} \\
=\frac{1}{\lambda_{i}-\lambda_{k}}\left(\frac{\left(x^{1}\right)^{2}}{a^{2}-\lambda_{i}}-\frac{\left(x^{1}\right)^{2}}{a^{2}-\lambda_{k}}+\frac{\left(x^{2}\right)^{2}}{b^{2}-\lambda_{i}}-\frac{\left(x^{2}\right)^{2}}{b^{2}-\lambda_{k}}+\frac{\left(x^{3}\right)^{2}}{c^{2}-\lambda_{i}}-\frac{\left(x^{3}\right)^{2}}{c^{2}-\lambda_{k}}\right) \\
=\frac{1}{\lambda_{i}-\lambda_{k}}\left(g\left(\lambda_{i}\right)-g\left(\lambda_{k}\right)\right)=0 .
\end{array}
$$

Thus the equations in (7.1) define a triple orthogonal system (Figure 7.1).

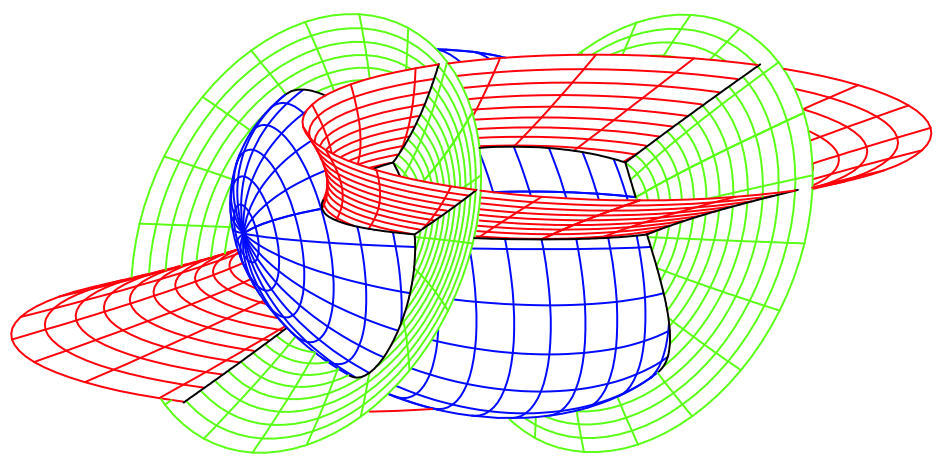

FIG. 7.1: A triple orthogonal system of ellipsoids and hyperboloids of one and two sheets

We now apply Lemma 7.1 and Dupin's theorem to find the lines of curvature on ellipsoids and hyperboloids of one and two sheets.

Example 7.1. Let the point $\left(x^{1}, x^{2}, x^{3}\right) \in \mathbb{R}^{3} \backslash\{0\}$ be given. We put

$$
\phi(\lambda)=\left(a^{2}-\lambda\right)\left(b^{2}-\lambda\right)\left(c^{2}-\lambda\right) g(\lambda)
$$

where $g$ is the function defined in (7.1). Thus we have

$$
\begin{aligned}
\phi(\lambda)= & \left(x^{1}\right)^{2}\left(b^{2}-\lambda\right)\left(c^{2}-\lambda\right)+\left(x^{2}\right)^{2}\left(a^{2}-\lambda\right)\left(c^{2}-\lambda\right)+ \\
& \left(x^{3}\right)^{2}\left(a^{2}-\lambda\right)\left(b^{2}-\lambda\right)-\left(a^{2}-\lambda\right)\left(b^{2}-\lambda\right)\left(c^{2}-\lambda\right)
\end{aligned}
$$

and $\phi(\lambda)=0$ is a cubic equation with zeros $\lambda_{k}(k=1,2,3)$, where

$$
\lambda_{1}<a^{2}<\lambda_{2}<b^{2}<\lambda_{3}<c^{2} .
$$

Consequently we may write

$$
\phi(\lambda)=\left(\lambda-\lambda_{1}\right)\left(\lambda-\lambda_{2}\right)\left(\lambda-\lambda_{3}\right) .
$$


We choose $\lambda=a^{2}$, and obtain from (7.3) and (7.5)

$$
\phi\left(a^{2}\right)=\left(x^{1}\right)^{2}\left(b^{2}-a^{2}\right)\left(c^{2}-a^{2}\right)=\left(a^{2}-\lambda_{1}\right)\left(a^{2}-\lambda_{2}\right)\left(a^{2}-\lambda_{3}\right),
$$

hence

$$
\left(x^{1}\right)^{2}=\frac{\left(a^{2}-\lambda_{1}\right)\left(a^{2}-\lambda_{2}\right)\left(a^{2}-\lambda_{3}\right)}{\left(a^{2}-b^{2}\right)\left(a^{2}-c^{2}\right)}
$$

and similarly

$$
\begin{aligned}
& \left(x^{2}\right)^{2}=\frac{\left(b^{2}-\lambda_{1}\right)\left(b^{2}-\lambda_{2}\right)\left(b^{2}-\lambda_{3}\right)}{\left(b^{2}-a^{2}\right)\left(b^{2}-c^{2}\right)}, \\
& \left(x^{3}\right)^{2}=\frac{\left(c^{2}-\lambda_{1}\right)\left(c^{2}-\lambda_{2}\right)\left(c^{2}-\lambda_{3}\right)}{\left(c^{2}-a^{2}\right)\left(c^{2}-b^{2}\right)} .
\end{aligned}
$$

If we choose $\lambda_{i}=$ const, then the identities in (7.6), (7.7) and (7.8) yield a parametrization of the surface given by the equation $g\left(\lambda_{i}\right)=0$ with respect to the values $\lambda_{j}$ and $\lambda_{k}$. We put

$$
\begin{gathered}
\alpha=a^{2}-\lambda_{i}, \quad \beta=b^{2}-\lambda_{i}, \quad \gamma=c^{2}-\lambda_{i}, \\
u^{1}=\lambda_{j}-\lambda_{i} \text { and } u^{2}=\lambda_{k}-\lambda_{i} .
\end{gathered}
$$

Then the surface given by the equation

$$
\frac{\left(x^{1}\right)^{2}}{\alpha}+\frac{\left(x^{2}\right)^{2}}{\beta}+\frac{\left(x^{3}\right)^{2}}{\gamma}=1
$$

has parametric representation

$$
\left\{\begin{array}{l}
x^{1}\left(u^{i}\right)= \pm \sqrt{\frac{\alpha\left(\alpha-u^{1}\right)\left(\alpha-u^{2}\right)}{(\alpha-\beta)(\alpha-\gamma)}}, \\
x^{2}\left(u^{i}\right)= \pm \sqrt{\frac{\beta\left(\beta-u^{1}\right)\left(\beta-u^{2}\right)}{(\beta-\alpha)(\beta-\gamma)}}, \\
x^{3}\left(u^{i}\right)= \pm \sqrt{\frac{\gamma\left(\gamma-u^{1}\right)\left(\gamma-u^{2}\right)}{(\gamma-\alpha)(\gamma-\beta)}} .
\end{array}\right\}
$$

The parameter lines with respect to this parametric representation are lines of curvature (Figures 7.2 and 7.3). 


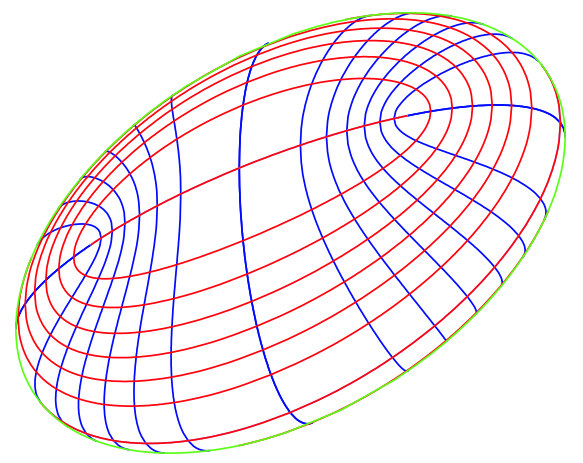

FIG. 7.2: Lines of curvature on an ellipsoid
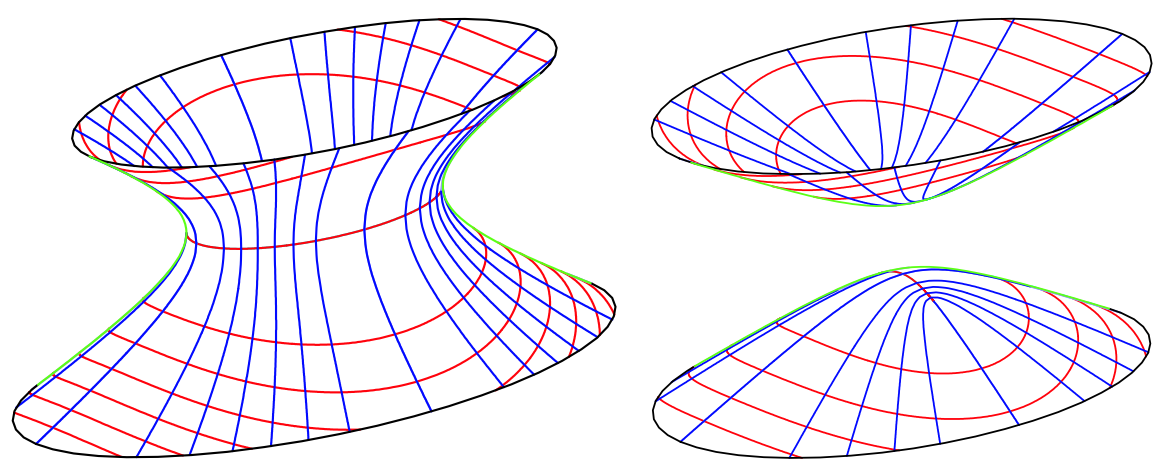

FIG. 7.3: Left: Lines of curvature on a hyperboloid of one sheet; Right: Lines of curvature on a hyperboloid of two sheets

\section{REFEREN CES}

1. L. BiARD, R.T. FAROUKI, N. SzAFRAn: Construction of rational surface patches bounded by lines of curvature. Computer Aided Geometric Design, 27(5), (2010), 359-371.

2. W.J. Che, J.C. PAul, X.P. Zhang: Lines of curvature and umbilical points for implicit surfaces. Computer Aided Geometric Design, 24(7) (2007) 395409.

3. K. ENDL and R. ENDL: Computergraphik 1 - Eine Software zur Geomerie in Turbo-Pascal. Würfel-Verlag, Biebertal-Vetzberg, 1989.

4. K. EndL and R. EndL: Computergraphik 2 - Eine Software zur Geomerie objektorientierter Programmierung mit Turbo-Pascal. Würfel-Verlag, BiebertalVetzberg, 1991.

5. M. FAILING: Entwicklung numerischer Algorithmen zur computergrafischen Darstellung spezieller Probleme der Differentialgeometrie und Kristallografie. $\mathrm{Ph}$. D. Thesis, Giessen, Shaker Verlag Aachen, 1996. 
6. M. FAILING and E. MALKOWSKY: Ein effizienter Nullstellenalgorithmus zur computergrafischen Darstellung spezieller Kurven und Flächen. Mitt. Math. Sem. Giessen 229 (1996), 11-28.

7. W.X. Huang, H.J.L. Wu, G.J. Wang: Constructing PDE-based surfaces bounded by geodesics or lines of curvature. Computers and Mathematics with Applications, 65(4), (2013), 673-681.

8. H.K. Joo, T. Yazaki, M. Takezawa, T. Maekawa: Differential geometry properties of lines of curvature of parametric surfaces and their visualization. Graphical Models 76 (2014), 224238.

9. E. Kalogerakis, D. Nowrouzezahrai, P. Simari, K. Singh: Extracting lines of curvature from noisy point clouds. Computer Aided Design, 41(4) (2009), 282292.

10. D. Laugwitz: Differentialgeometrie. B. G. Teubner Verlag, 1970.

11. C.Y. LI, R.H. WANG, C.G. ZHU: An approach for designing a developable surface through a given line of curvature. Computer Aided Design 45(3) (2013) 621627.

12. E. MALKOWSKY: An open software in OOP for computer graphics and some applications in differential geometry. Proceedings of the 20th South African Symposium on Numerical Mathematics, (1994) 51-80

13. E. Malkowsky: Visualisation and animation in mathematics and physics. Proceedings of the Institute of Mathematics of NAS of Ukraine (50)(3) (2004), 14151422.

14. E. Malkowsky and W. Nickel: Computergrafik in der Differentialgeometrie. Vieweg-Verlag, Braunschweig, 1993.

15. E. Malkowsky, F. Özger and V. Veličković: Some Spaces Related to Cesaro Sequence Spaces and an Application to Crystallography. MATCH Commun. Math. Comput. Chem., 70(3) (2013), 867-884.

16. E. Malkowsky and V. VeličKović: Analytic transformations between surfaces with animations. Proceedings of the Institute of Mathematics of NAS of Ukraine (50)(3) (2004), 1496-1501.

17. E. MALKowsky and V. VeličKović: Topologies of some new sequence spaces, their duals, and the graphical representations of neighborhoods. Topology and its Applications, 158(12) (2011), 1369-1380.

18. E. Malkowsky and V. VeličKović: Some New Sequence Spaces, Their Duals and a Connection with Wulff's Crystal. MATCH Commun. Math. Comput. Chem., 67(3) (2012), 589-607.

19. E. Malkowsky and V. VeličKović: The duals of certain matrix domains of factorable triangles and some related visualisations. Filomat, 27(5) (2013), 821829.

20. J. Sotomayor, R. Garcia: Lines of Curvature on Surfaces, Historical Comments and Recent Developments. São Paulo Journal of Mathematical Sciences 2(1) (2008), 99143

21. V. VeličKović: Vizualizacija u matematici pomoću objektno orijentisanog programskog paketa za linijsku grafiku. Ph. D. Thesis, University of Niš, 2012

22. X.P. Zhang, W.J. Che, J.C. Paul: Computing lines of curvature for implicit surfaces. Computer Aided Geometric Design, 26(9) (2009), 923-940. 
Vesna Veličković

University of Niš

Faculty of Sciences and Mathematics

Department of Computer Science

Višegradska 33

$18000 \mathrm{Niš}$, Serbia

vesna@pmf.ni.ac.rs 